\title{
A Pleomorphic Rhabdomyosarcoma Mimicking an Inguinal Hernia: A Case Report and Review of the Literature
}

\author{
Anna Koumarianou $^{a}$ Sofia Karageorgopoulou $^{a}$ \\ Anastasios Machairas $^{b} \quad$ Theodore Liakakos $^{b}$ Stavroula Chranioti $^{c}$ \\ George Dimitriadis $^{a}$ Evangelos P. Misiakos ${ }^{b}$ \\ ${ }^{a}$ Section of Medical Oncology, Second Department of Propaedeutic Internal Medicine, and \\ ${ }^{\mathrm{b}}$ Third Department of Surgery, Attikon University Hospital, and ' Department of Pathology, \\ Asklipieion Voulas General Hospital, Athens, Greece
}

\section{Key Words}

Rhabdomyosarcoma $\cdot$ Inguinal tumour $\cdot$ Chemotherapy

\begin{abstract}
A 59-year-old male presented with a painful right inguinal swelling and deep vein thrombosis at the ipsilateral leg. An inguinal hernia was initially diagnosed, but during surgery a large mass was found anteriorly to the peritoneal sheaths. Histology revealed a high-grade pleomorphic rhabdomyosarcoma. The mass advanced rapidly, occupying the whole right iliac fossa and metastasizing to the lung. Despite first- and second-line chemotherapy, the patient deteriorated rapidly and died. Rhabdomyosarcomas should be managed in specialized centres as they have prognostic factors and histologic features still controversial and poorly clarified.
\end{abstract}

\section{Introduction}

Rhabdomyosarcoma (RMS) is the most common soft tissue sarcoma in children, representing the fifth most frequent solid tumour in childhood [1]. However, it is an extremely rare tumour in adults, accounting for less than $1 \%$ of malignancies in this population [2].

The pleomorphic subtype of RMS is histologically diagnosed in $19 \%$ of adult cases and is characterized by aggressive biology and a significantly worse prognosis compared to the other histological subtypes [3]. 
The aim of the present study is to report a very rare case of inguinal pleomorphic RMS and review the related literature.

\section{Case Report}

A 59-year-old male presented to the emergency department of our hospital with a painful hard swelling at the right inguinal fossa. His medical history included a mitral valve prolapse with low blood pressure and a 4-month history of deep vein thrombosis of the right lower leg. He also reported a significant weakness and a weight loss of $4 \mathrm{~kg}$ during the last 2 months. The laboratory tests revealed a mild normochromic, normocytic anaemia (Hb: $10 \mathrm{~g} / \mathrm{dl}$ ), slightly elevated serum calcium levels (10.6 mg/dl; normal value range: $8.2-10.4 \mathrm{mg} / \mathrm{dl})$ and increased serum lactate dehydrogenase levels (576 U/l; normal value range: $230-460 \mathrm{U} / \mathrm{l}$ ). Based on a surgical consultation, this inguinal mass was considered a complicated right inguinal hernia, and the patient was admitted for surgical management. Surprisingly, the operation revealed an inoperable mass anterior to the peritoneal sheaths, and a biopsy was taken for histological examination.

The macroscopic examination reported a firm mass measuring $1.6 \times 1.2 \times 0.8 \mathrm{~cm}$ with grayish white cut surface. Analysis of the haematoxylin-eosin-stained sections revealed a malignant pleomorphic mesenchymal neoplasm, characterized mainly by proliferation of atypical spindle and few epithelioid cells, in a plexiform pattern, admixed with giant multinucleated cells with abundant eosinophilic nucleoli (fig. 1). Immunohistochemistry displayed strong and diffuse positive stain s for smooth muscle actin, vimentin, and CD99, and focally positive nuclear and cytoplasmic stains for Myo D1 and myogenin (fig. 2). Some cells were positive for desmin, and $60 \%$ of the tumour cells expressed MDM-2 protein. Ki-67 (MIB-1) ranged from 50-60\%. The combination of the above findings supported the diagnosis of a high-grade pleomorphic RMS.

The tumour staging, using abdominal and chest CT scan, revealed enlarged right common iliac and external iliac lymph nodes, invading the right obturator internus muscle and measuring up to $8.7 \mathrm{~cm}$ in diameter, with central hypodense areas consisting of necrotic foci. A 3-cm secondary lesion was found at the posterior right lower pulmonary lobe. A bone scan was negative for bone involvement but showed marked radionuclide uptake at the periphery of the inguinal mass. The bone marrow biopsy was negative for bone marrow infiltration. The patient's neoplasm was staged as group IV according to the International RMS Study Group (IRSG) surgical-pathologic grouping system, and as stage 4 according to the IRSG staging system (table 1 and table 2) [4].

One month after his admission, the patient was referred to the oncology department. However, his performance status had already deteriorated to 2 due to the rapid growth of the right pelvic mass (12 and $19 \mathrm{~cm}$ in the transverse and the longitudinal diameter, respectively) causing considerable oedema and leg pain. The patient required strong opioid analgesics for pain relief.

Chemotherapy was immediately administered using the VAC regimen (vincristine, actinomycin-D, and cyclophosphamide) in 4 cycles. Re-evaluation by CT scans revealed that the disease had further advanced to the lung and the pelvis, and new secondary lesions were identified in the liver. A secondline chemotherapy was administered (ifosfamide and etoposide) in 3 cycles. However, the patient's symptoms continued to deteriorate, although there was no evidence of significant toxicity from the treatment. The whole situation was thoroughly discussed with the patient and his family, and the decision was to discontinue chemotherapy and administer only symptomatic treatment. Ultimately, the patient passed away, 10 months following the initial diagnosis.

\section{Discussion}

This case represents the second inguinal pleomorphic RMS ever reported in the international literature [5]. RMSs are a group of different pathobiologic entities linked by a common characteristic: to form neoplastic skeletal muscle. This is derived from biological forces related to aberrant transcription signals that lead to the production of 
myogenic proteins. These differing morphologic features were recognized in the mid1900s by Horn and Enterline, who divided RMSs into embryonal, alveolar, botryoid, and pleomorphic subtypes [6]. However, recent histologic and molecular studies led to the description of additional entities, such as spindle cell and anaplastic RMSs.

Following further refinements of the original entities, using molecular biology, the International Classification of Rhabdomyosarcomas separated the histological subtypes according to their prognostic significance [6]. As a consequence, botryoid and spindle cell RMSs were assigned to the superior-prognosis group, embryonal RMSs to the intermediate-prognosis group, and alveolar RMSs to the poor-prognosis group. However, pleomorphic RMSs were not included in this classification, as they concerned the paediatric population in which the pleomorphic subtype is a very rare entity $[3,7]$.

Pleomorphic RMSs occur predominately in middle-aged men (median age: 54-56 years) and are mostly located in the lower extremity. Less frequent sites of presentation are the abdomen, retroperitoneum, chest wall, spermatic cord/testes, upper extremity, mouth, and orbit $[8,9]$. They typically have an aggressive clinical course, demonstrating an overall poor prognosis $[8,9]$.

Given the rarity of the disease and the lack of large prospective controlled studies, very few related data are available, deriving from studies with small numbers of patients. Furlong et al. [8] reported 38 pleomorphic RMS cases (28 men and 10 women). These patients presented with a rapidly growing mass (with a median size of $6.9 \mathrm{~cm}$ ) involving mainly the lower extremity. The response to treatment was rather poor as approximately $70 \%$ of the patients died of the disease within 20 months. The progression-free survival of $45 \%$ of these patients was reported to be 9 months. Gaffney et al. [9] reported 11 pleomorphic RMS cases (10 men and 1 woman; median age: 56 years) with the thigh being the most common site of disease presentation. From the 8 cases that had clinical follow-up, 1 patient was alive 20 months after the initial diagnosis, whereas the remaining 7 patients had died 2-28 months following diagnosis.

In the study by Ferrari et al. [2], localized pleomorphic RMS was found in 37 patients ( $20 \%$ of the total RMS population), and complete surgical resection was possible for $74 \%$ of these patients (compared to 30\% for other RMS histologic subtypes). Only 12 patients received chemotherapy. Overall, the 5 -year event-free survival and overall survival rates were 29.9 and $53.4 \%$ for the pleomorphic RMSs compared to 32.9 and $45.7 \%$, respectively, for the non-pleomorphic RMSs. However, these results correspond to the localized rather than the metastatic setting, which was the case in our patient. In the same study, it has been suggested that when the paediatric treatment guidelines are followed in adult patients, the response rates are comparable to those observed in children. According to the major conclusions derived from the meta-analysis of the Intergroup RMS Studies I-IV, VAC remains the standard regimen for patients with advanced disease [4].

The underlying aetiology of the poor prognosis in this disease is not yet well understood. Reports regarding prognostic and predictive factors of adult pleomorphic RMS patients are very few and contradictory. Little et al. [10] suggest that pleomorphic RMS is the most common RMS subtype in adults, accounting for $43 \%$ of the RMS population with locoregional disease. Pleomorphic RMSs were grouped and analyzed together with embryonal RMSs (a total of 63 patients) and did not show significant differences in terms of progression-free survival or overall survival compared to the 
alveolar subtype. Nevertheless, metastasis-free survival was slightly higher for the pleomorphic/embryonal group (58 vs. $32 \%$ for the alveolar group, $p=0.04$ ). In another study, Joshi et al. [11] reported that increasing age is a negative prognostic factor among children with RMSs.

On the other hand, a retrospective analysis from the Surveillance, Epidemiology and End Results database of 205 adults and 17 children with pleomorphic RMSs, which was recently published by Sultan et al. [3], concluded that adult patients with pleomorphic RMSs demonstrate a worse prognosis, and that this is irrespective of the site of origin of the tumour or the histological subtype. It is noteworthy that there were exceptionally few children with pleomorphic RMSs in this database, and that these had a risk to die twice as high as adults (HR 3.73 vs.1.79, $\mathrm{p}<0.001$ ).

\section{Conclusion}

In accordance with previous studies, our patient had a pleomorphic RMS with highly malignant features, progressing very quickly and not responding to aggressive chemotherapy. These findings further support our previous suggestion that these patients should be managed in specialized centres in order to receive optimal treatment and participate in large, well-designed, prospective trials to analyze prognostic factors and histological features that are still controversial.

Table 1. According to the IRSG, RMSs are classified into 4 post-surgical pathologic groups, depending on the extent of the residual disease after initial surgical treatment

\begin{tabular}{ll} 
Group & Definition \\
\hline I & $\begin{array}{l}\text { Localized tumour, completely removed with pathologically } \\
\text { clear margins and no regional lymph node involvement }\end{array}$ \\
\hline II & $\begin{array}{l}\text { Localized tumour, grossly removed with (a) microscopically } \\
\text { involved margins, (b) involved, grossly resected regional } \\
\text { lymph nodes }\end{array}$ \\
\hline III $\quad \begin{array}{l}\text { Localized tumour, with gross residual disease after grossly } \\
\text { incomplete removal, or biopsy only }\end{array}$ \\
\hline IV $\quad$ Distant metastases present at diagnosis \\
\hline
\end{tabular}


Table 2. The pre-clinical IRSG staging system was adopted during the fourth IRS (1991-1997) and classifies patients according to the site of tumour origin, size, regional nodal involvement and presence or absence of metastatic disease

\begin{tabular}{lllll}
\hline Stage & Site of primary tumour & $\begin{array}{l}\text { Tumour size } \\
\mathrm{cm}\end{array}$ & $\begin{array}{l}\text { Regional } \\
\text { lymph nodes }\end{array}$ & $\begin{array}{l}\text { Distant } \\
\text { metastases }\end{array}$ \\
\hline 1 & $\begin{array}{l}\text { Orbit, } \\
\text { non-parameningeal head and neck, } \\
\text { GU non-bladder/prostate } \\
\text { biliary tract }\end{array}$ & Any size & N0, N1 & M0 \\
\hline 2 & All other sites & $\leq 5$ & N0 & M0 \\
\hline 3 & All other sites & $\leq 5 ;$ & N1; & M0 \\
\hline 4 & Any site & $>5$ & N0, N1 & N1 \\
\hline
\end{tabular}

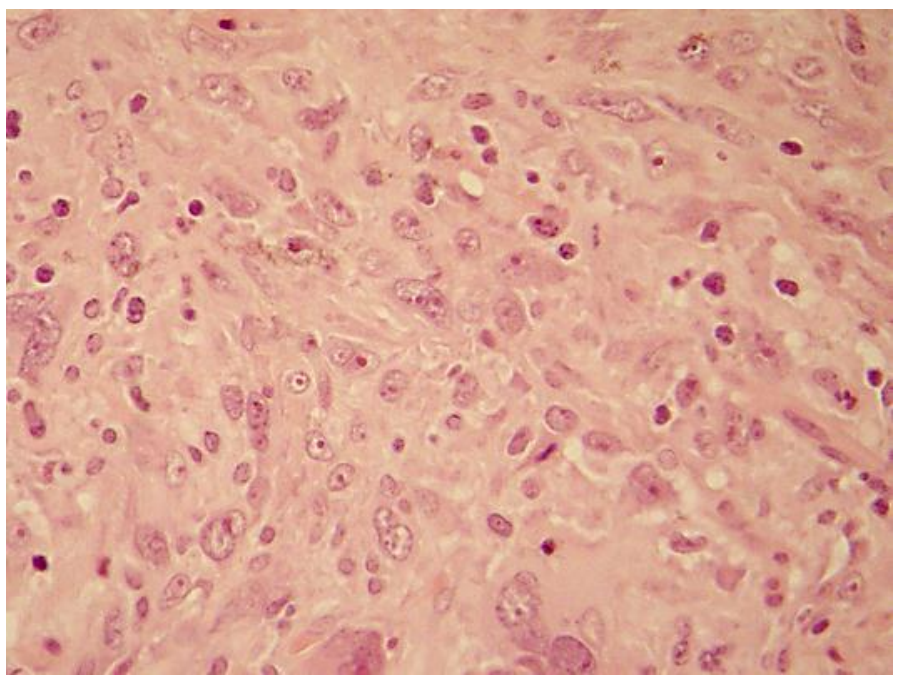

Fig. 1. Malignant mesenchymal neoplasm with atypical spindle and epithelioid cells; a large number of them present with abundant eosinophilic nucleoli. Haematoxylin-eosin stain. $\times 400$. 


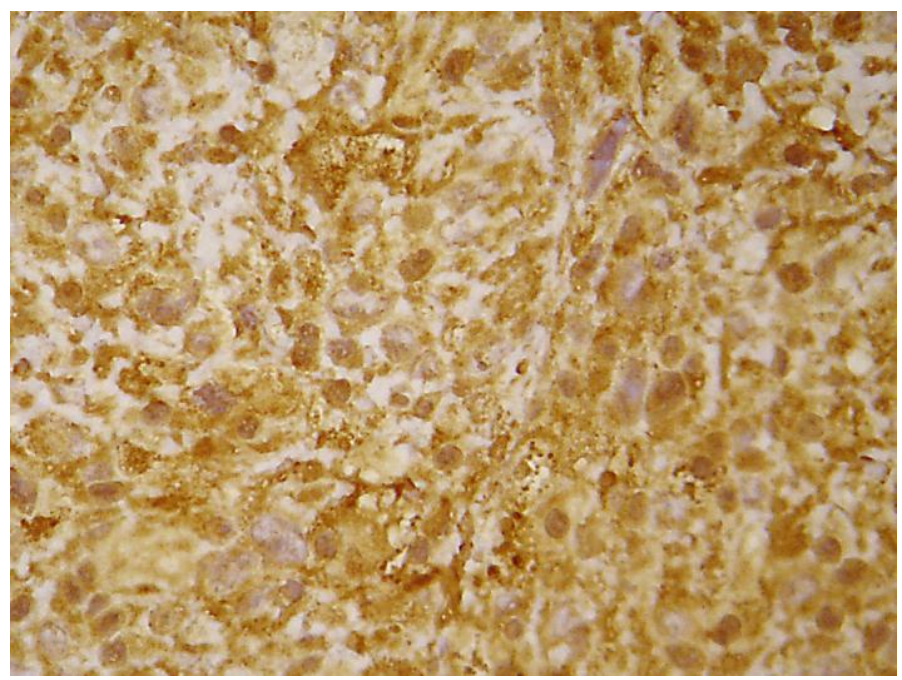

Fig. 2. Positive nuclear and cytoplasmic stain for myogenin in neoplasmatic cells. $\times 400$.

\section{References}

1 Simon JH, Paulino AC, Ritchie JM, Mayr NA, Buatti JM: Presentation, prognostic factors and patterns of failure in adult rhabdomyosarcoma. Sarcoma 2003;7:1-7.

-2 Ferrari A, Dileo P, Casanova M, Bertuli R, Gandola L, Navarria P, Collini P, Gronchi A, Olmi P, FossatiBellani F, Casali PG: Rhabdomyosarcoma in adults. A retrospective analysis of 171 patients treated at a single institution. Cancer 2003;98:571-580.

-3 Sultan I, Qaddoumi I, Yaser S, Rodriguez-Galindo C, Ferrari A: Comparing adult and paediatric rhabdomyosarcoma in the surveillance, epidemiology and end results program, 1973 to 2005 an analysis of 2,600 patients. J Clin Oncol 2009;27:3391-3397.

-4 Raney RB, Maurer HM, Anderson JR, Andrassy RJ, Donaldson SS, Qualman ST, Wharam MD, Wiener ES, Crist WM: The Intergroup Rhabdomyosarcoma Study Group (IRSG): major lessons from the IRS-I through IRS-IV studies as background for the current IRS-V treatment protocols. Sarcoma 2001;5:9-15.

-5 Eyden B: Pleomorphic rhabdomyosarcoma showing smooth-muscle and fibrohistiocytic differentiation: a single case report. Ultrastruct Pathol 2010;34:42-47.

6 Parham DM, Ellison DA: Rhabdomyosarcomas in adults and children: an update. Arch Pathol Lab Med 2006;130:1454-1465.

7 Newton WA Jr, Soule EH, Hamoudi AB, Reiman HM, Shimada H, Beltangady, Maurer H: Histopathology of childhood sarcomas, Intergroup Rhabdomyosarcoma Studies I and II: clinicopathologic correlation. J Clin Oncol 1988;6:67-75.

-8 Furlong MA, Mentzel T and Fanburg-Smith JC: Pleomorphic rhabdomyosarcoma in adults: a clinicopathologic study of 38 cases with emphasis on morphologic variants and recent skeletal musclespecific markers. Mod Pathol 2001;4:595-603.

-9 Gaffney EF, Dervan PA, Fletcher CD: Pleomorphic rhabdomyosarcoma in adulthood. Analysis of 11 cases with definition of diagnostic criteria. Am J Surg Pathol 1993;17:601-609.

10 Little DJ, Ballo MT, Zagars GK, Pisters PW, Patel SR, El-Naggar AK, Garden AS, Benjamin RS: Adult rhabdomyosarcoma: outcome following multimodality treatment. Cancer 2002;95:377-388.

-11 Joshi D, Anderson JR, Paidas C, Breneman J, Parham DM, Crist W: Age is an independent prognostic factor in rhabdomyosarcoma: a report from the Soft Tissue Sarcoma Committee of the Children's Oncology Group. Pediatr Blood Cancer 2004;42:64-73. 